\title{
Preventing chronic disease and mortality among primary care patients with mental disorders
}

\author{
Isabelle A. Vallerand
}

University of Calgary

\section{Primary care goals and challenges}

Primary care practices represent the first point of entry into the health care system, and offer services in disease prevention, health promotion, health maintenance, diagnosis and treatment of acute and chronic conditions. ${ }^{1}$ The need to receive quality comprehensive and continued care may be especially important for patients with a multitude of comorbid chronic conditions. In particular, managing mental illness represents a difficult public health challenge given its association with additional chronic diseases and excess mortality. For instance, people with depression have been shown to have an excess rate of mortality due to cardiovascular disease, cerebrovascular disease, respiratory diseases, endocrine and metabolic diseases and accidents or intoxications. ${ }^{2}$ Since individuals experiencing symptoms of mental illness often first seek treatment in primary care, these practices offer an ideal venue for implementing strategies to prevent or reduce the burden of chronic diseases and premature mortality among patients with mental disorders. ${ }^{3}$ Yet, barriers to the monitoring and management of patients with mental disorders and comorbid physical conditions, such as a tendency to focus solely on treating mental rather than physical health concerns may be contributing to the large gap that exists in the coordination of physical and mental health care today. ${ }^{4,5}$

Strategies to prevent chronic disease and mortality in patients with mental disorders

While there are many risk factors and causes of morbidity and mortality that could be targeted for prevention, the question of course is what can be done and where should efforts begin? From a primary care and public health standpoint, perhaps the most important risk factors to target are the ones that can be considered modifiable. The importance of being able to identify a modifiable risk factor lies in being able to exert control over a risk factor, which should thereby result in a reduction of the risk of chronic disease or mortality. Mental illness is often thought to be associated with a detrimental lifestyle and risky behaviours, ${ }^{6}$ which can theoretically be changed. Strategies targeting modifiable risk factors that have been shown to be associated with mental disorders such as (but not limited to) smoking, ${ }^{7}$ alcohol misuse ${ }^{8}$ and obesity ${ }^{9}$ are likely to align well with the goals of primary care in terms of improving the overall health of the patient.

Concurrently, it is imperative to consider the timing and context in delivering lifestyle-based interventions relative to the course of the mental illness. For instance, some patients with acute mental disturbances may need to be stabilized first before embarking on interventions aimed at quitting smoking, reducing alcohol consumption and attempting to lose weight. Though there exists some evidence to date that interventions targeting modifiable risk factors may be effective among patients with mental disorders, concerns have been raised regarding the sustainability of these effects. One factor that may compromise intervention efforts by primary care providers is that risk factors for chronic disease and mortality among patients with mental disorders (e.g. smoking, alcohol misuse and obesity) are closely tied to a patient's social network, thus rendering these unhealthy behaviours self-perpetuating. ${ }^{6}$ In primary care patients without mental disorders, smoking cessation can be difficult to achieve and maintain, thus one would expect that these challenges be further amplified in the context of patients with mental disorders who have a host of other risk factors and chronic diseases simultaneously. One potential strategy may be to prevent chronic disease by first identifying people with mental disorders in primary care and maintain a focus on stabilizing the mental disorder first before shifting efforts to continuing care, which may be necessary in order to tackle complex lifestyle changes. 
In doing so, this could help to make these patients more similar to the general population, which may be less challenging to treat by primary care providers in the long run. With the expectation that eventually once or if a mental disorder is successfully treated, the strategies based on modifiable lifestyle risk factors would continue to offer benefits to the overall health of the patient (i.e. both physical and mental health) in terms of reduced risk of developing chronic diseases and premature mortality.

\section{Future directions}

This commentary aimed to shed light on some lifestylebased strategies (e.g. smoking cessation, alcohol reduction, and weight-loss) for reducing chronic disease and excess mortality among patients with mental disorders in primary care. Additional considerations for primary care providers may entail decisions on when to initiate these strategies along the course of mental illness, by balancing the need to stabilize mental conditions as well as managing physical health. Future primary care research is needed to determine the long-term impact of the proposed strategies on mortality among patients with mental disorders that may help to bridge the gap between physical and mental health.

\section{References}

1. Canada H. About primary health care [internet]. 2012 [updated 2012 Aug 23; cited 2014 Apr 25]. Available from: http://www.hc-sc.gc.ca/hcs-sss/ prim/about-apropos-eng.php.

2. Laursen TM, Munk-Olsen T, Nordentoft M, Mortensen PB. Increased mortality among patients admitted with major psychiatric disorders: A register-based study comparing mortality in unipolar depressive disorder, bipolar affective disorder, schizoaffective disorder, and schizophrenia. J Clin Psychiat. 2007;68(6):899-907.

3. Reilly S, Planner C, Hann M, Reeves D, Nazareth I, Lester H. The role of primary care in service provision for people with severe mental illness in the United Kingdom. PLOS ONE. 2012;7(5):e36468.

4. De Hert M, Cohen DAN, Bobes J, Cetkovich-Bakmas M, Leucht S, Ndetei DM, et al. Physical illness in patients with severe mental disorders. II. Barriers to care, monitoring and treatment guidelines, plus recommendations at the system and individual level. World Psychiatry. 2011;10(2):138-51.

5. Colton CW, Manderscheid RW. Congruencies in increased mortality rates, years of potential life lost, and causes of death among public mental health clients in eight states. Preventing chronic disease. 2006;3(2):A42.

6. Druss BG, Bornemann TH. Improving health and health care for persons with serious mental illness: The window for us federal policy change. JAMA. 2010;303(19):1972-3.

7. Brown $\mathrm{S}$, Kim M, Mitchell C, Inskip $\mathrm{H}$. Twenty-five year mortality of a community cohort with schizophrenia. Brit J Psychiat. 2010;196(2):116-21.

8. Pompili M, Serafini G, Innamorati M, Dominici G, Ferracuti S, Kotzalidis GD, et al. Suicidal behavior and alcohol abuse. Int J Environ Res Public Health. 2010;7(4):1392-431

9. Daumit GL, Dickerson FB, Wang NY, Dalcin A, Jerome GJ, Anderson CA, et al. A behavioral weight-loss intervention in persons with serious mental illness. NEJM. 2013;368(17):1594-602.

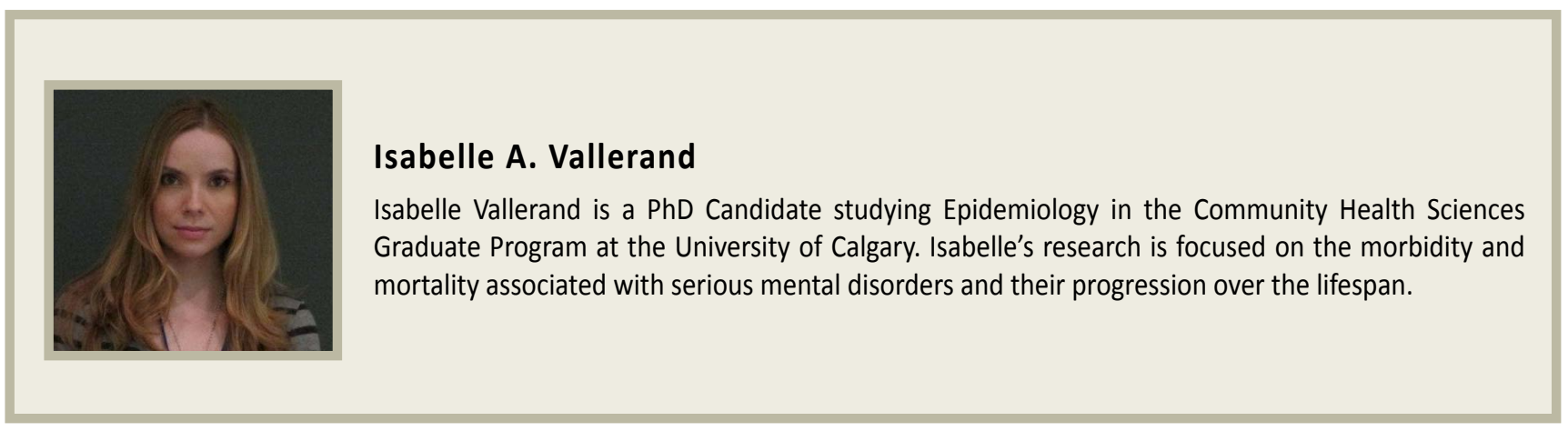

